\title{
Systemic inflammation response index predicts prognosis in patients with clear cell renal cell carcinoma: a propensity score-matched analysis
}

This article was published in the following Dove Medical Press journal: Cancer Management and Research

\author{
Zhen Chen* \\ Kai Wang* \\ Hao Lu \\ Dong Xue \\ Min Fan \\ Qianfeng Zhuang \\ Shuai Yin \\ Xiaozhou He \\ Renfang Xu
}

Department of Urology, The Third Affiliated Hospital of Soochow University, Changzhou 213003,

People's Republic of China

*These authors contributed equally to this work
Correspondence: Xiaozhou He;

Renfang Xu

Department of Urology, The Third

Affiliated Hospital of Soochow University,

I85 Juqian Street, Changzhou 213003,

People's Republic of China

Tel +86 5 I9 6887 I252;

+865196887 I25।

Email xiaozhouhel23@I63.com;

xurenfangcz@163.com
Purpose: In the present study, we aimed to evaluate the prognostic significance of the systemic inflammation response index (SIRI), which was defined based on peripheral blood counts of neutrophils, lymphocytes, and monocytes, in patients with localized or locally advanced clear cell renal cell carcinoma (CCRCC).

Patients and methods: The prognostic value of SIRI was evaluated in a primary cohort consisting of 414 patients with localized or locally advanced CCRCC and then further validated in an independent cohort composed of 168 patients.

Results: Kaplan-Meier survival analyses of both cohorts revealed that CCRCC patients with high SIRI levels exhibited poorer overall survival (OS) and cancer-specific survival (CSS) compared with those with low SIRI levels. Furthermore, univariate and multivariate analyses identified SIRI as a significant independent predictor for both OS (HR: 4.853; 95\% CI: 2.362-9.972; $P<0.001$ ) and CSS (HR: 5.913; 95\% CI: 2.681-13.040; $P<0.001$ ). Following propensity score matching analysis, SIRI remained an excellent predictor for both OS and CSS. The area under the curve for SIRI was larger than that of the platelet-to-lymphocyte ratio (PLR), neutrophil-tolymphocyte ratio (NLR), monocyte-to-lymphocyte ratio (MLR), and Memorial Sloan Kettering Cancer Center (MSKCC) prognostic score in both cohorts.

Conclusion: SIRI might be a better prognostic predictor than PLR, NLR, MLR, and MSKCC score in patients with localized or locally advanced CCRCC.

Institutional review board approval number: (2010) Scientific Research Project No. 39 Keywords: systemic inflammation response index, prognosis, clear cell renal cell carcinoma, PSM

\section{Introduction}

Renal cell carcinoma (RCC) represents $2 \%-3 \%$ of all adult malignancies, among which a large proportion of RCC cases are pathologically categorized as clear cell renal cell carcinoma (CCRCC). ${ }^{1,2}$ Currently, radical nephrectomy is the optimal therapy for localized or locally advanced RCC, since RCC is not sensitive to chemoradiotherapy. However, a recurrence rate of $20 \%-30 \%$ is observed after resection during follow-up. ${ }^{3,4}$ To evaluate the postoperative risks of RCC patients and to optimize individualized therapy, the seventh edition of the American Joint Committee on Cancer (AJCC) TNM staging system is frequently referenced as a clinical guideline to predict the outcomes of RCC patients. ${ }^{5}$ Disappointingly, the clinical outcomes greatly vary among patients who are classified into the same TNM stage and have received similar therapy. ${ }^{6}$ Therefore, it is urgently necessary to identify other reliable prognostic factors in order to better 
assess the outcomes of patients with localized or locally advanced RCC.

Cancer-related inflammation, mainly including the local immune response and systemic inflammation, is a defined hallmark of malignant tumors, which greatly contributes to tumorigenesis and development of cancer. ${ }^{7}$ Briefly, peripheral blood leukocytes, including neutrophils, lymphocytes, and monocytes, are involved in systemic inflammation. Increasing evidence has suggested that there is complex interplay between leukocytes and the prognosis of patients with various types of cancers, including RCC. ${ }^{8-10}$ The neutrophil-tolymphocyte ratio (NLR), platelet-to-lymphocyte ratio (PLR), and monocyte-to-lymphocyte ratio (MLR), ${ }^{11-13}$ which are derived from peripheral blood leukocyte counts, have been recognized as reliable parameters.

Recently, a newly named inflammatory indicator, the systemic inflammation response index (SIRI), which is mainly defined based on peripheral neutrophil, lymphocyte and monocyte counts, has been determined to be a reliable prognostic factor in pancreatic cancer. ${ }^{14}$ However, its potential prognostic value in RCC patients remains largely unexplored. Therefore, in the present study, we aimed to investigate the prognostic value of SIRI in patients with localized or locally advanced CCRCC who received radical or partial nephrectomy. Furthermore, the prognostic value of SIRI was compared with that of NLR, PLR, MLR, and MSKCC score. In addition, the Cox proportional hazards model and propensity score matching (PSM) analysis were used to enhance the statistical reliability and determine the potential prognostic value of SIRI.

\section{Materials and methods \\ Patients}

Written informed consent was obtained from every participant enrolled in the study. Current study was carried out in accordance with the Declaration of Helsinki, and the study protocol was approved by the Ethics Committee of the Third Affiliated Hospital of Soochow University. The patients who received radical or partial nephrectomy at the Third Affiliated Hospital of Soochow University (Changzhou, China) from January 2003 to December 2013 were retrospectively identified. To guarantee the accuracy and objectivity of the data collected, patients were excluded if they had a history of antitumor therapy, other concurrent tumors, other acute or chronic concurrent noncancer diseases (including liver disease, inflammation, and infection), and concurrent distant metastasis, or those were lost during follow-up. Finally, a total of 582 eligible patients were enrolled in the present study, among which 168 patients were randomly assigned to the external validation cohort, and all other patients were assigned to the primary cohort. For patients with locally advanced CCRCC, postoperative follow-up procedures were performed every 6 months during the first 3 years and annually thereafter. For patients with localized CCRCC, follow-up procedures included imaging every 6 months in the first year and annually thereafter. Neither neoadjuvant nor adjuvant therapy was performed on the enrolled patients.

\section{Data collection}

The following clinicopathological data were collected for each enrolled patient, including age at surgery, sex, T stage, $\mathrm{N}$ stage, Fuhrman grade, and whether tumor necrosis and lymphovascular invasion were observed. The TNM stage was assigned based on the 2010 AJCC TNM classification. ${ }^{5}$ The definition of tumor necrosis was coagulative necrosis as determined by a microscope. ${ }^{15}$ Lymphovascular invasion was defined as the spread of cancer cells to blood vessels or lymphatic vessels but not the underlying muscular walls. ${ }^{16}$ Data on relevant laboratory indicators, including neutrophil counts and lymphocyte counts, were collected 1 week before surgery. All the above-mentioned information was retrospectively extracted from the Third Affiliated Hospital of Soochow University. The SIRI was calculated according to the following formula: SIRI $=$ monocyte $\times$ neutrophil $/$ lymphocyte. NLR was the ratio of neutrophils to lymphocytes. ${ }^{11}$ MLR was the ratio of monocytes to lymphocytes. ${ }^{12}$ PLR was the ratio of platelets to lymphocytes. ${ }^{17}$ The optimal cutoff values, including SIRI $(\leq 1.35,>1.35)$, NLR $(\leq 2.17$, $>2.17)$, PLR $(\leq 179.83,>179.83)$, and $\operatorname{MLR}(\leq 0.30,>0.30)$, were determined by using X-tile software (http://www.tissuearray.org/rimmlab). ${ }^{18}$

\section{Statistical analyses}

Statistical analyses were conducted as previously described. ${ }^{19}$ Briefly, statistical tests were performed using SPSS Statistics software (SPSS 22.0, Chicago, IL, USA) and MedCalc software (Version 11.4.2.0, MedCalc, Inc., Belgium). Two-sided $P$-values $<0.05$ were considered as statistically significant. Overall survival (OS) rate was determined from KaplanMeier curves and compared to detect statistically significant differences using the log-rank test. A nearest-neighbor matching algorithm was employed in the PSM analysis, with a maximal difference between propensity scores under $30 \%$ of the propensity score SD. The Mantel-Cox regression methodology was used for the univariate analysis. Multivariable analyses using Cox proportional hazards models 
included variables with $P<0.05$ from the univariate analyses. The discriminatory abilities of SIRI, NLR, PLR, MLR, and MSKCC score in evaluating prognosis were assessed by comparing the area under the curve (AUC) values of the receiver operating characteristic (ROC) curves. Differences were compared with MedCalc software to confirm whether they were statistically significant.

\section{Results}

\section{Patient characteristics}

Table 1 summarizes the clinicopathological characteristics of patients in the primary and validation cohorts. Of the 414 patients in the primary cohort, 257 (62.1\%) were males and $152(36.7 \%)$ were 60 years of age or older. The median age was 56.3 years, ranging from 24 to 80 years. The median follow-up period was 69.2 months, ranging from 1 to 151 months. At the final follow-up, 50 (12.1\%) patients passed away, and 364 (87.9\%) patients survived.

In the validation cohort, there were 168 patients, including $101(60.1 \%)$ males and $65(38.7 \%)$ patients who were 60 years of age or older. At the final follow-up, 26 (15.5\%) patients passed away and $142(84.5 \%)$ patients survived.

\section{Cutoff values for continuous variables}

Table 1 presents the cutoff values for all continuous variables. The patients were divided into two groups based on the optimal cutoff value of SIRI $(\leq 1.35, \mathrm{n}=299 ;>1.35, \mathrm{n}=115)$. The optimal cutoff values for NLR, MLR, and PLR were $2.17,0.30$, and 179.83, respectively. The cutoff value of $\mathrm{Hb}$ was $130 \mathrm{~g} / \mathrm{L}$ for males and $115 \mathrm{~g} / \mathrm{L}$ for females (lower limit of the reference). The cutoff value of ALP was $125 \mathrm{U} / \mathrm{L}$ (upper limit of the reference). The cutoff value of lactate dehydrogenase $(\mathrm{LDH})$ was $1.5 \times 245 \mathrm{U} / \mathrm{L}$ (1.5 $\times$ upper limit of the reference).

\section{Associations between the SIRI and clinicopathological characteristics of the primary patient cohort}

Table 2 summarizes the associations between the SIRI and clinicopathological characteristics in patients of the primary cohort. Higher SIRI levels were significantly associated with older age at surgery $(P=0.001)$, male gender $(P=0.001)$, more advanced T stage $(P<0.001)$, more advanced $\mathrm{N}$ stage $(P=0.021)$, higher Fuhrman grade $(P=0.001)$, larger tumor size $(P=0.038)$, tumor necrosis $(P<0.001)$, lower $\mathrm{Hb}$ level $(P=0.001)$, and elevated ALP $(P=0.032)$ and LDH $(P=0.009)$ levels.
Table I Characteristics of primary and validation cohorts

\begin{tabular}{|c|c|c|}
\hline Variables & $\begin{array}{l}\text { Primary cohort } \\
(n=4 \mid 4)\end{array}$ & $\begin{array}{l}\text { Validation cohort } \\
(n=168)\end{array}$ \\
\hline \multicolumn{3}{|l|}{ Age (years) } \\
\hline$\leq 60$ & 262 (63.3\%) & 103 (61.3\%) \\
\hline$>60$ & $152(36.7 \%)$ & 65 (38.7\%) \\
\hline \multicolumn{3}{|l|}{ Sex } \\
\hline Male & 257 (62.1\%) & 101 (60.1\%) \\
\hline Female & 157 (37.9\%) & 67 (39.9\%) \\
\hline \multicolumn{3}{|l|}{ T stage } \\
\hline TI & 338 (8I.6\%) & I38 (82.1\%) \\
\hline $\mathrm{T} 2$ & $44(10.6 \%)$ & 15 (8.9\%) \\
\hline T3 & $32(7.7 \%)$ & $15(8.9 \%)$ \\
\hline \multicolumn{3}{|l|}{$N$ stage } \\
\hline No & 404 (97.6\%) & 164 (97.6\%) \\
\hline $\mathrm{NI}$ & $10(2.4 \%)$ & $4(2.4 \%)$ \\
\hline \multicolumn{3}{|c|}{ Fuhrman grade } \\
\hline I & $86(20.8 \%)$ & $3 \mathrm{I}(18.5 \%)$ \\
\hline 2 & $199(48.1 \%)$ & 77 (45.8\%) \\
\hline 3 & 94 (22.7\%) & $48(28.6 \%)$ \\
\hline 4 & $22(5.3 \%)$ & $9(5.4 \%)$ \\
\hline \multicolumn{3}{|c|}{ Tumor size $(\mathrm{cm})$} \\
\hline$\leq 5$ & $264(63.8 \%)$ & III (66.1\%) \\
\hline$>5$ & I 48 (35.7\%) & $56(33.3 \%)$ \\
\hline \multicolumn{3}{|c|}{ Tumor necrosis } \\
\hline Absent & 375 (90.6\%) & 155 (92.3\%) \\
\hline Present & $39(9.4 \%)$ & $13(7.7 \%)$ \\
\hline \multicolumn{3}{|l|}{ LVI } \\
\hline Absent & 393 (94.9\%) & 157 (93.5\%) \\
\hline Present & $21(5.1 \%)$ & II (6.5\%) \\
\hline \multicolumn{3}{|c|}{ Hemoglobin $(g / L)$} \\
\hline$\leq \mathrm{LLN}$ & 49 (II.8\%) & $23(13.7 \%)$ \\
\hline$>$ LLN & 365 (88.2\%) & 145 (86.3\%) \\
\hline \multicolumn{3}{|l|}{ ALP } \\
\hline$\leq \mathrm{ULN}$ & 395 (95.4\%) & I6I (95.8\%) \\
\hline$>$ ULN & 15 (3.6\%) & $7(4.2 \%)$ \\
\hline \multicolumn{3}{|l|}{$\mathrm{LDH}$} \\
\hline$\leq 1.5 \times \mathrm{ULN}$ & 403 (97.3\%) & 166 (98.8\%) \\
\hline$>1.5 \times \mathrm{ULN}$ & $7(1.7 \%)$ & $2(1.2 \%)$ \\
\hline \multicolumn{3}{|l|}{ SIRI } \\
\hline$\leq 1.35$ & $299(72.2)$ & $121(72.0)$ \\
\hline$>1.35$ & II5 (27.8) & $47(28.0)$ \\
\hline \multicolumn{3}{|l|}{ NLR } \\
\hline$\leq 2.17$ & $214(51.7 \%)$ & $87(5 \mathrm{I} .8 \%)$ \\
\hline$>2.17$ & 200 (48.3\%) & 81 (48.2\%) \\
\hline$\leq 0.30$ & 274 (66.2\%) & 109 (64.9\%) \\
\hline$>0.30$ & 140 (33.8\%) & 59 (35.1\%) \\
\hline \multicolumn{3}{|l|}{ PLR } \\
\hline$\leq 179.83$ & 356 (86.0\%) & I47 (87.5\%) \\
\hline$>179.83$ & $58(\mathrm{I} 4.0 \%)$ & $2 \mathrm{I}(12.5 \%)$ \\
\hline \multicolumn{3}{|l|}{ MSKCC score } \\
\hline $\mathrm{I}$ & $311(75.1 \%)$ & 118 (70.2\%) \\
\hline 2 & $91(22.0 \%)$ & $47(28.0 \%)$ \\
\hline 3 & $3(2.9 \%)$ & $3(1.8 \%)$ \\
\hline
\end{tabular}

Abbreviations: LDH, lactate dehydrogenase; LLN, lower limit of normal; LVI, lymphovascular invasion; MLR, monocyte-to-lymphocyte ratio; MSKCC, Memorial Sloan Kettering Cancer Center; NLR, neutrophil-to-lymphocyte ratio; PLR, plateletto-lymphocyte ratio; SIRI, systemic inflammation response index; ULN, upper limit of normal. 


\section{Prognostic value of SIRI}

Patients with higher SIRI values $(>1.35)$ had a significantly worse OS $(P<0.001)$ and cancer-specific survival (CSS; $P<0.001)$ than those with lower SIRI values $(\leq 1.35)$ in the primary cohort before PSM analysis (Figure 1A, B). Since there were some differences in clinicopathological characteristics between the low SIRI and high SIRI groups, PSM analysis was utilized to minimize these discriminations. During such process, 97 patients selected from the low SIRI group were respectively paired with high SIRI patients using the nearest-neighbor algorithm. After PSM analysis, the differences in clinicopathological characteristics were well balanced and evenly distributed in the low SIRI group and high SIRI group (all $P>0.05$; Table 2). Kaplan-Meier survival curves confirmed that patients in high SIRI group had worse OS and CSS (Figure 1C, D) after PSM analysis. In the validation cohort, the same conclusion was drawn from the Kaplan-Meier curves (Figure 1E, F).

Table 3 shows the outcomes of the multivariate analysis for OS and CSS before PSM analysis in the primary cohort. Considering the original correlations among SIRI, NLR, MLR, and PLR, multivariate analysis was conducted on the above-mentioned inflammation-based prognostic scores respectively, indicating that SIRI ( $\mathrm{HR}=4.853 ; 95 \% \mathrm{CI}$ : 2.362-9.972; $P<0.001)$, NLR $(P=0.001)$, MLR $(P=0.001)$, and T stage $(P=0.011)$ were significantly and independently associated with OS. We found similar results for CSS in the multivariate analysis.

The prognostic significance of the preoperative SIRI was further elucidated in subgroups of TNM stage (I+II/III+IV) and Fuhrman grade $(\mathrm{G} 1+\mathrm{G} 2 / \mathrm{G} 3+\mathrm{G} 4)$. Patients with high SIRI levels had a significantly shortened OS and CSS compared with those with low SIRI levels in the TNM stage I+II subgroup (OS, $P<0.001$, Figure $2 \mathrm{~A}$; CSS, $P<0.001$, Figure 2B), TNM stage III+IV subgroup (OS, $P=0.004$, Figure 2C; CSS, $P=0.004$, Figure 2D), Fuhrman grade G1+G2 subgroup (OS, $P=0.005$, Figure 2E; CSS, $P=0.001$, Figure $2 \mathrm{~F}$ ), and Fuhrman grade G3+G4 subgroup (OS, $P<0.001$, Figure 2G; CSS, $P<0.001$, Figure $2 \mathrm{H}$ ).

\section{Comparison of the discriminatory abilities of SIRI, NLR, PLR, MLR, and MSKCC score}

To compare the discriminatory power of SIRI, NLR, PLR, MLR, and MSKCC score, we generated ROC curves for OS at the 3-and 5-year follow-up examinations. Figure 3 shows that the AUC value was higher for SIRI compared with NLR, PLR,
MLR, and MSKCC score at both the 3- and 5-year follow-up in both the primary cohort and validation cohort, and such difference was only significant for some of the prognostic scores. Table 4 illustrates the discriminatory ability comparisons of SIRI with NLR, PLR, MLR, and MSKCC score.

\section{Discussion}

The potentially substantial connection between cancer and inflammation was originally proposed by Virchow in the 19th century. ${ }^{20}$ Gradually, the critical role of inflammation in mediating tumorigenesis, progression, and metastasis of malignancies has become widely recognized. ${ }^{10,21-23}$ Systemic inflammatory indexes, including NLR, PLR, and MLR, have been shown to predict prognosis of various cancers, including RCC. ${ }^{11-13}$ A newly named inflammatory marker, SIRI, which is defined based on peripheral blood counts of neutrophils, monocytes, and lymphocytes, has been found to be an independent risk factor for survival among patients with pancreatic cancer. SIRI is considered to be better than MLR and NLR in terms of predictive accuracy, and it is able to reflect the status of local immune response and systemic inflammation in patients with pancreatic cancer. ${ }^{14}$

In the present study, both the Cox proportional hazards model and PSM analysis were applied to confirm the independent prognostic value of SIRI for OS and CSS among patients with localized or locally advanced CCRCC in two independent cohorts. The predictive ability of SIRI was more powerful compared with NLR, PLR, and MLR. To date, the TNM staging system and Fuhrman grading system have been widely used in clinical practice to predict prognosis of RCC patients. However, it is still difficult to predict outcomes for patients with early-stage or low-grade RCC. In the present study, the patients were further stratified for subgroup analysis based on their TNM stage and Fuhrman grade. We found that the prognostic value of SIRI remained powerful for early-stage (TNM stage I+II; Figure 2A, B) and low-grade (Fuhrman G1+ G2; Figure 2E, F) CCRCC patients. Taken together, our results indicated that SIRI could predict OS for CCRCC patients, even for patients with early-stage or low Fuhrman grade disease. The powerful prognostic value of SIRI in those early-stage or low-grade subgroups suggested that it could be used to identify patients with a high risk of recurrence or metastasis, to whom adjuvant therapy should be preferably administered. Meanwhile, standard and advanced laboratory evaluations of peripheral neutrophil, monocyte, and lymphocyte counts have contributed to the accuracy, accessibility, and regular utilization of SIRI in clinical practice. Therefore, these results were crucially valuable for 

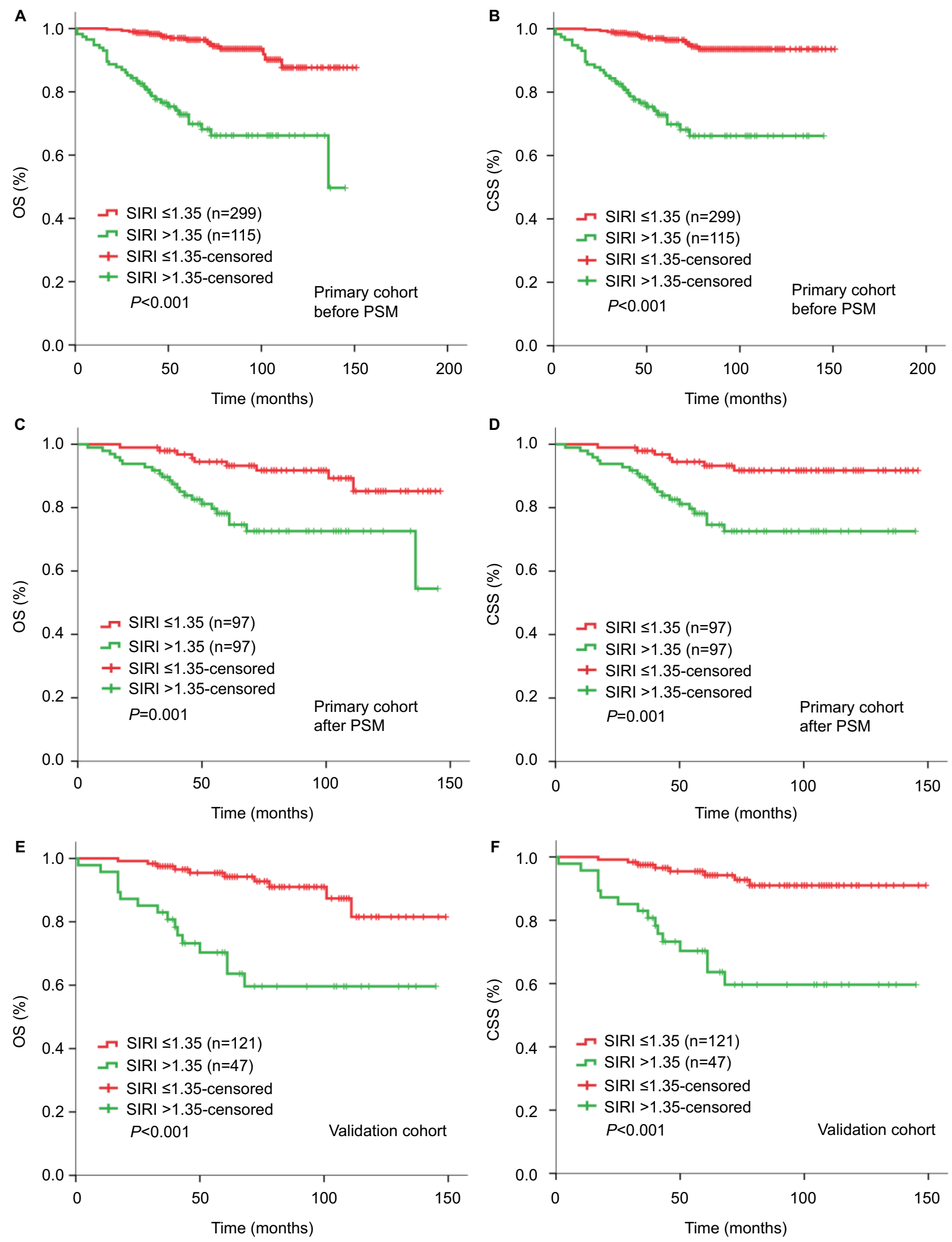

Figure I Kaplan-Meier curves for OS and CSS according to SIRI in the primary cohort before PSM analysis (A, B), after PSM analysis (C, D), and in the validation cohort (E, F).

Abbreviations: CSS, cancer-specific survival; OS, overall survival; PSM, propensity score matching; SIRI, systemic inflammation response index. 
Table 2 Associations between SIRI and clinicopathological characteristics in primary cohort before and after PSM analysis

\begin{tabular}{|c|c|c|c|c|c|c|}
\hline \multirow[t]{2}{*}{ Variables } & \multicolumn{3}{|l|}{ Pre-PSM } & \multicolumn{3}{|l|}{ Post-PSM } \\
\hline & $\begin{array}{l}\text { SIRI } \leq I .35 \\
(n=299)\end{array}$ & $\begin{array}{l}\text { SIRI }>I .35 \\
(n=|| 5)\end{array}$ & $P$-value & $\begin{array}{l}\text { SIRI } \leq I .35 \\
(n=97)\end{array}$ & $\begin{array}{l}\text { SIRI >I.35 } \\
(n=97)\end{array}$ & $P$-value \\
\hline Age (years) & & & $0.001 *$ & & & 1 \\
\hline$\leq 60$ & 204 (68.2\%) & 58 (50.4\%) & & $49(50.5 \%)$ & 49 (50.5\%) & \\
\hline$>60$ & 95 (3I.8\%) & 57 (49.6\%) & & 48 (49.5\%) & 48 (49.5\%) & \\
\hline Sex & & & $0.00 I^{*}$ & & & 0.226 \\
\hline Male & I7I (57.2\%) & 86 (74.8\%) & & 79 (8I.4\%) & 72 (74.2\%) & \\
\hline Female & $128(42.8 \%)$ & $29(25.2 \%)$ & & $18(18.6 \%)$ & $25(25.8 \%)$ & \\
\hline T stage & & & $<0.00 I^{*}$ & & & 0.066 \\
\hline TI & 260 (87.0\%) & 78 (67.8\%) & & $86(88.7 \%)$ & $74(76.3 \%)$ & \\
\hline $\mathrm{T} 2$ & $26(8.7 \%)$ & $18(15.7 \%)$ & & $6(6.2 \%)$ & 15 (I5.5\%) & \\
\hline T3 & $13(4.3 \%)$ & $19(16.5 \%)$ & & $5(5.2 \%)$ & $8(8.2 \%)$ & \\
\hline $\mathrm{N}$ stage & & & $0.021^{*}$ & & & \\
\hline No & 295 (98.7\%) & $109(94.8 \%)$ & & 93 (95.9\%) & 94 (96.9\%) & \\
\hline $\mathrm{NI}$ & $4(1.3 \%)$ & $6(5.2 \%)$ & & $4(4.1 \%)$ & $3(3.1 \%)$ & 0.7 \\
\hline Fuhrman grade & & & $0.00 I^{*}$ & & & 0.814 \\
\hline$I$ & $69(23.8 \%)$ & 17 (I5.3\%) & & $17(17.5 \%)$ & $15(15.5 \%)$ & \\
\hline 2 & $143(49.3 \%)$ & 56 (50.5\%) & & 49 (50.5\%) & $53(54.6 \%)$ & \\
\hline 3 & $70(24.1 \%)$ & $24(21.6 \%)$ & & $26(26.8 \%)$ & $22(22.7 \%)$ & \\
\hline 4 & $8(2.8 \%)$ & $14(12.6 \%)$ & & $5(5.2 \%)$ & $7(7.2 \%)$ & \\
\hline Tumor size $(\mathrm{cm})$ & & & $0.038^{*}$ & & & 0.373 \\
\hline$\leq 5$ & 200 (67.1\%) & 64 (56.1\%) & & 64 (66.0\%) & $58(59.8 \%)$ & \\
\hline$>5$ & $98(32.9 \%)$ & 50 (43.9\%) & & $33(34.0 \%)$ & $39(40.2 \%)$ & \\
\hline Tumor necrosis & & & $<0.00 I^{*}$ & & & 0.426 \\
\hline Absent & 281 (94.0\%) & 94 (8I.7\%) & & $84(86.6 \%)$ & $84(86.6 \%)$ & \\
\hline Present & $18(6.0 \%)$ & $21(18.3 \%)$ & & $13(13.4 \%)$ & $13(13.4 \%)$ & \\
\hline LVI & & & 0.113 & & & 0.7 \\
\hline Absent & 287 (96.0\%) & 106 (92.2\%) & & 94 (96.9\%) & $93(95.9 \%)$ & \\
\hline Present & $12(4.0 \%)$ & $9(7.8 \%)$ & & $3(3.1 \%)$ & $4(4.1 \%)$ & \\
\hline Hemoglobin $(\mathrm{g} / \mathrm{L})$ & & & $0.00 I^{*}$ & & & 0.557 \\
\hline$\leq$ LLN & 26 (8.7\%) & $23(20.0 \%)$ & & $17(17.5 \%)$ & $14(14.4 \%)$ & \\
\hline$>$ LLN & 273 (91.3\%) & 92 (80.0\%) & & 80 (82.5\%) & $83(85.6 \%)$ & \\
\hline ALP & & & $0.023^{*}$ & & & 0.7 \\
\hline$\leq \mathrm{ULN}$ & 290 (97.6\%) & 105 (92.9\%) & & 94 (96.9\%) & 93 (95.9\%) & \\
\hline$>$ ULN & $7(2.4 \%)$ & $8(7.1 \%)$ & & $3(3.1 \%)$ & $4(4.1 \%)$ & \\
\hline $\mathrm{LDH}$ & & & $0.009 *$ & & & 1 \\
\hline$\leq 1.5 \times$ ULN & 295 (99.3\%) & $108(95.6 \%)$ & & $96(99.0 \%)$ & 96 (99.0\%) & \\
\hline$>1.5 \times$ ULN & $2(0.7 \%)$ & 5 (4.4\%) & & $\mathrm{I}(\mathrm{I} .0 \%)$ & $\mathrm{I}(\mathrm{I} .0 \%)$ & \\
\hline
\end{tabular}

Notes: $* P<0.05$.

Abbreviations: LDH, lactate dehydrogenase; LLN, lower limit of normal; LVI, lymphovascular invasion; PSM, propensity score matching; SIRI, systemic inflammation response index; ULN, upper limit of normal.

increasing the accuracy of the established prognostic factors, demonstrating that SIRI might serve as an effective method for accurate diagnosis and early decision-making for patients with localized or locally advanced CCRCC after radical or partial nephrectomy.

Accumulating evidence indicates that there is a complex interplay among systemic inflammation, tumorigenesis, and tumor progression. Studies have shown that the systemic inflammatory response destroys the host immune response, facilitating the escape of tumor cells from immune surveillance, inhibiting apoptosis and promoting genomic instability, angiogenesis, invasion, and metastasis in cancer patients. ${ }^{10,20,24}$ Peripheral blood leukocytes, including neutrophils, lymphocytes, and monocytes, are involved in the systemic inflammatory response and participate in the process of tumorigenesis and cancer progression. First, neutrophils can be evoked by cancer-related inflammatory chemokines and cytokines, such as IL- 6 and tumor necrosis factor. In addition, high levels of neutrophils promote the proliferation, invasion, and metastasis of cancer cells and 
Table 3 OS, CSS, and multivariate analyses in primary cohort before PSM analysis

\begin{tabular}{|c|c|c|c|c|}
\hline \multirow[t]{2}{*}{ Variables } & \multicolumn{2}{|c|}{ Multivariate analysis for OS } & \multicolumn{2}{|c|}{ Multivariate analysis for CSS } \\
\hline & HR (95\% Cl) & $P$-value & HR (95\% Cl) & $P$-value \\
\hline Age (years) & & $0.252^{\mathrm{a}}$ & & $0.429^{a}$ \\
\hline$>60$ years vs $\leq 60$ years & $1.506(0.748-3.033)$ & & $\mathrm{I} .352(0.64 \mathrm{I}-2.852)$ & \\
\hline \multicolumn{5}{|l|}{ Sex } \\
\hline \multicolumn{5}{|l|}{ Male vs Female } \\
\hline T stage & & $0.01 \mathrm{I}^{\mathrm{a}, *}$ & & $0.0 \mathrm{II} \mathrm{I}^{\mathrm{a},{ }^{*}}$ \\
\hline $\mathrm{TI}$ & Reference & & Reference & \\
\hline T2 & $1.7 \mid 0(0.7|7-4.08|)$ & $0.227^{\mathrm{a}}$ & $1.883(0.760-4.667)$ & $0.172^{\mathrm{a}}$ \\
\hline T3 & $5.212(1.770-15.345)$ & $0.003^{\mathrm{a},{ }^{*}}$ & $5.520(1.812-16.820)$ & $0.003^{\mathrm{a}, *}$ \\
\hline $\mathrm{N}$ stage & & $0.11 \mathrm{I}^{\mathrm{a}, *}$ & & $0.125^{\mathrm{a},{ }^{*}}$ \\
\hline NI vs No & $3.002(0.777-\mid$ I I.589) & & $2.935(0.742-1 \mathrm{I} .603)$ & \\
\hline Fuhrman grade & & $0.064^{\mathrm{a}}$ & & $0.104^{a}$ \\
\hline $\mathrm{I}$ & Reference & & Reference & \\
\hline 2 & $2.36 I(0.620-8.99 I)$ & $0.208^{\mathrm{a}}$ & $2.066(0.515-8.292)$ & $0.306^{\mathrm{a}}$ \\
\hline 3 & $3.444(0.90 \mathrm{I}-13.163)$ & $0.07 I^{\mathrm{a}}$ & $3.081(0.76 I-12.469)$ & $0.115^{\mathrm{a}}$ \\
\hline 4 & $0.738(0.140-3.879)$ & $0.720^{\mathrm{a}}$ & $0.692(0.129-3.7 / 2)$ & $0.667^{\mathrm{a}}$ \\
\hline \multicolumn{5}{|l|}{ Tumor size $(\mathrm{cm})$} \\
\hline$>5$ vs $\leq 5$ & $2.187(0.997-4.795)$ & $0.05 \mathrm{I}^{\mathrm{a}}$ & $2.576(1.098-6.047)$ & $0.030^{\mathrm{a}}$ \\
\hline Tumor necrosis & & $0.146^{\mathrm{a},{ }^{*}}$ & & $0.08 \mathrm{I}^{\mathrm{a}}$ \\
\hline Present vs absent & $1.730(0.826-3.624)$ & & $1.971(0.919-4.227)$ & \\
\hline LVI & & $0.124^{\mathrm{a}}$ & & $0.079^{a}$ \\
\hline Present vs absent & $2.354(0.79 I-7.006)$ & & $2.682(0.892-8.069)$ & \\
\hline Hemoglobin $(g / L)$ & & $0.075^{\mathrm{a}}$ & & $0.143^{\mathrm{a}}$ \\
\hline$\leq \mathrm{LLN}$ vs $>$ LLN & $2.133(0.928-4.906)$ & & $1.953(0.798-4.779)$ & \\
\hline ALP & & $0.986^{\mathrm{a}}$ & & $0.883^{\mathrm{a}}$ \\
\hline$>$ ULN vs $\leq$ ULN & $0.989(0.289-3.384)$ & & $0.909(0.254-3.25 \mathrm{I})$ & \\
\hline $\mathrm{LDH}$ & & $0.790^{\mathrm{a}}$ & & $0.814^{\mathrm{a}}$ \\
\hline$>1.5 \times$ ULN vs $\leq 1.5 \times U L N$ & $1.26 I(0.230-6.918)$ & & $1.226(0.226-6.649)$ & \\
\hline SIRI & & $<0.00 \mathrm{I}^{\mathrm{a},{ }^{*}}$ & & $<\left.0.00\right|^{\mathrm{a},{ }^{*}}$ \\
\hline$\leq 1.35$ vs $>1.35$ & $4.853(2.362-9.972)$ & & $5.913(2.68|-| 3.040)$ & \\
\hline NLR & & $0.00 \mathrm{I}^{\mathrm{b}, *}$ & & $0.00 \mathrm{I}^{\mathrm{b},{ }^{*}}$ \\
\hline$>2.17$ vs $\leq 2.17$ & $3.650(1.735-7.678)$ & & $4.192(1.852-9.49 \mid)$ & \\
\hline MLR & & $0.00 \mathrm{I}^{\mathrm{b}, *}$ & & $0.002^{\mathrm{b}, *}$ \\
\hline$>0.30$ vs $\leq 0.30$ & $3.417(1.674-6.972)$ & & $3.416(1.596-7.314)$ & \\
\hline PLR & & $0.913^{b}$ & & $0.78 \mathrm{I}^{\mathrm{b}}$ \\
\hline$>179.83$ vs $\leq 179.83$ & I.05I (0.434-2.543) & & I. $137(0.460-2.808)$ & \\
\hline
\end{tabular}

Notes: a Age, $\mathrm{T}$ stage, $\mathrm{N}$ stage, Fuhrman grade, tumor size, tumor necrosis, LVI, hemoglobin, ALP, LDH, and SIRI variables were tested in a multivariate analysis. ${ }^{b} \mathrm{NLR}$, MLR, and PLR were evaluated together with age, T stage, $\mathrm{N}$ stage, Fuhrman grade, tumor size, tumor necrosis, LVI, Hb, ALP, and LDH, respectively; and multivariate analysis for these inflammation-based prognostic scores was then conducted. $* P<0.05$

Abbreviations: CSS, cancer-specific survival; LDH, lactate dehydrogenase; LLN, lower limit of normal; LVI, lymphovascular invasion; MLR, monocyte-to-lymphocyte ratio; NLR, neutrophil-to-lymphocyte ratio; OS, overall survival; PLR, platelet-to-lymphocyte ratio; PSM, propensity score matching; SIRI, systemic inflammation response index; ULN, upper limit of normal.

induce resistance to cancer therapeutics. ${ }^{10,25,26}$ Second, the immune response to cancer cells in humans depends mainly on the level of lymphocytes, which can be sharply diminished by systemic inflammation. Relative lymphocytopenia may reflect a lower level of CD4+ T cells, which impairs cancer immune surveillance and defence. ${ }^{10,27}$ Third, tumor-associated macrophages (TAMs), which are derived from circulating monocytes, can damage the immune system and promote invasion, proliferation, angiogenesis, and metastasis of cancer cells. ${ }^{10,28}$ To some extent, the level of monocytes in the peripheral blood can reflect the levels of TAMs. Therefore, a high level of monocytes represents a high tumor burden in cancer patients. ${ }^{29}$ Moreover, cancer-associated inflammation leads to an elevated SIRI and promotes tumorigenesis and cancer progression, eventually resulting in an unfavorable outcome in cancer patients.

There were several possible limitations in the present study. First, this was a retrospective, single-institute study, and selection biases might exist during data collection. Therefore, PSM analysis was performed to minimize the 

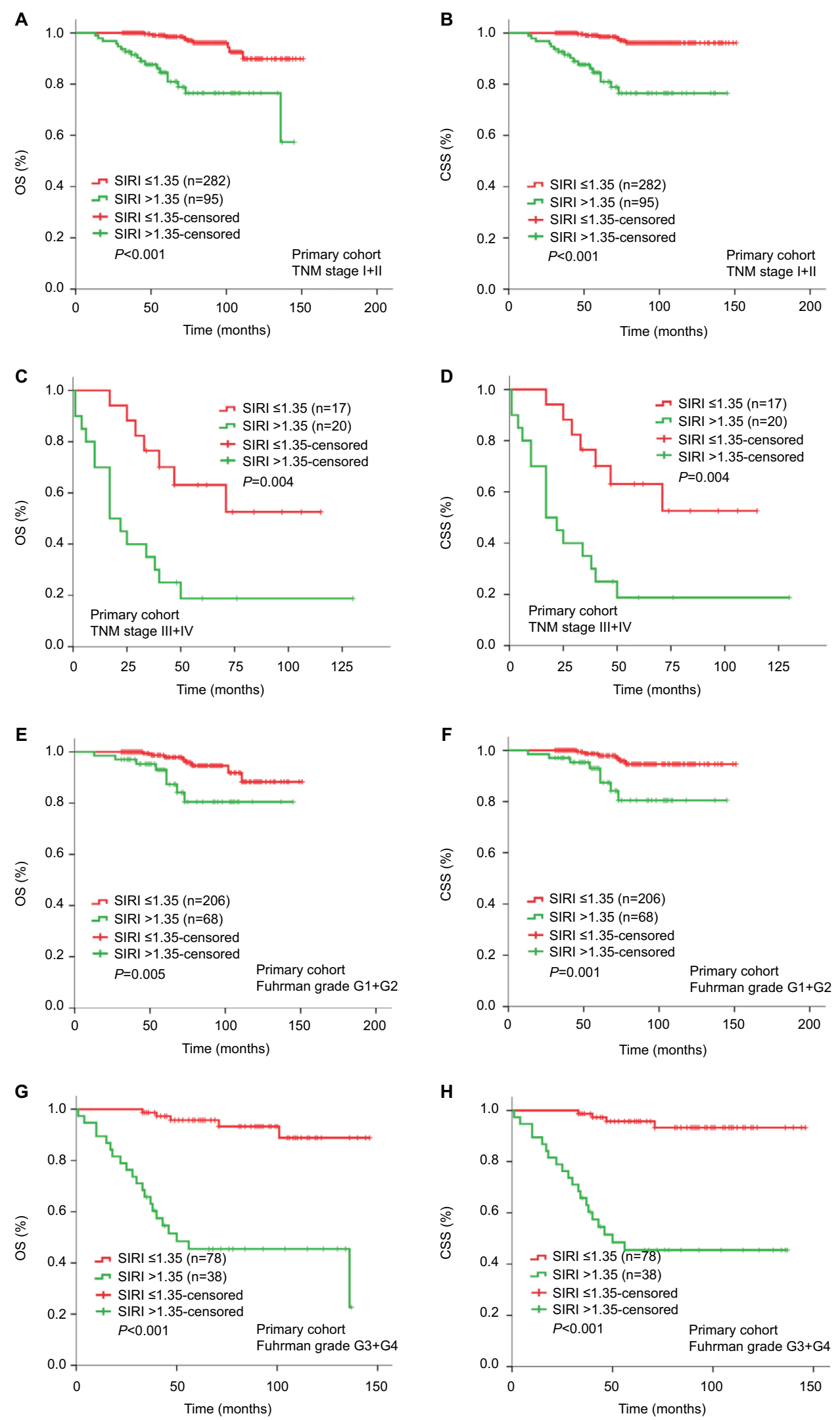

Figure 2 Kaplan-Meier curves for OS and CSS according to SIRI in the TNM stage I+II subgroup (A, B), TNM stage III+IV subgroup (C, D), Fuhrman grade GI+G2 subgroup (E, F), and Fuhrman grade $\mathrm{G} 3+\mathrm{G} 4$ subgroup $(\mathbf{G}, \mathbf{H})$ in the primary cohort.

Abbreviations: CSS, cancer-specific survival; OS, overall survival; SIRI, systemic inflammation response index. 
A

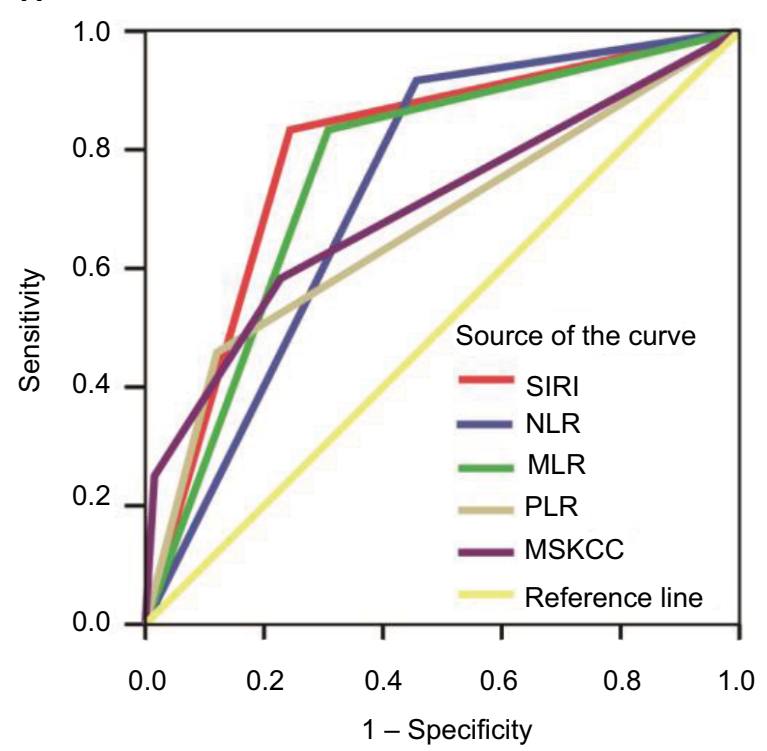

C

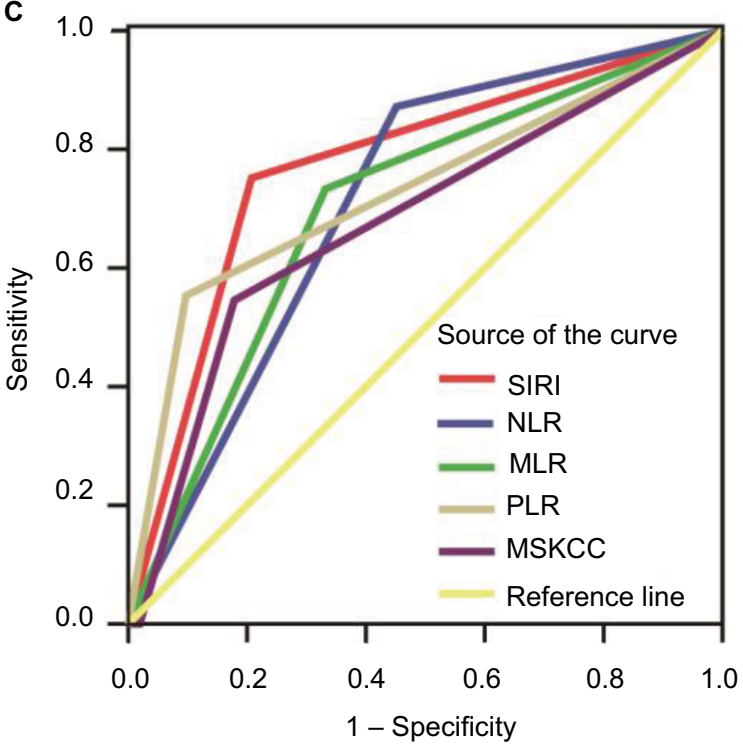

B

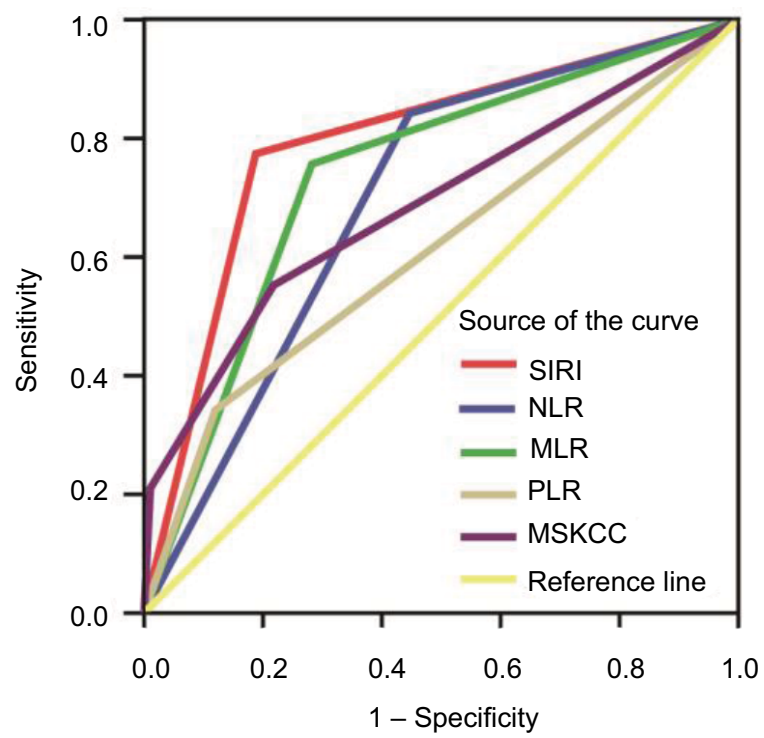

D

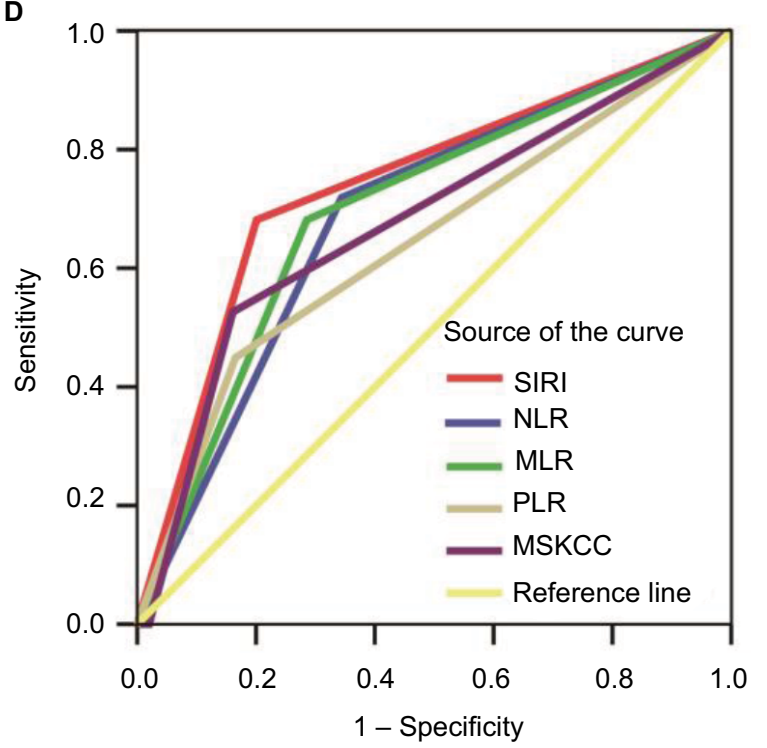

Figure 3 Predictive ability of SIRI was compared with NLR, PLR, MLR, and MSKCC score by ROC curves at 3 and 5 years of follow-up in the primary (A, B) and validation (C, D) cohorts.

Abbreviations: CSS, cancer-specific survival; MLR, monocyte-to-lymphocyte ratio; MSKCC, Memorial Sloan Kettering Cancer Center; NLR, neutrophil-to-lymphocyte ratio; PLR, platelet-to-lymphocyte ratio; ROC, receiver operating characteristic; SIRI, systemic inflammation response index.

discriminations caused by the baseline information of different groups. However, a multicenter, prospective study is necessary to confirm our findings. Another limitation was the lack of certain follow-up information, such as relapse-free survival and progression-free survival, although OS and CSS already indicated the prognostic value of SIRI in CCRCC.

\section{Conclusion}

In the present study, we established a novel and easily assessable prognostic scoring system, named SIRI, which was constructed based on pretreatment levels of peripheral neutrophils, lymphocytes, and monocytes. Collectively, our findings suggested that SIRI might serve as an independent prognostic predictor, and it could be used to better predict the prognosis in patients with localized or locally advanced CCRCC.

\section{Acknowledgment}

This work was financially supported by the National Science Foundation of Jiangsu Province (Grant no. BK20141161 and BK20150251). 
Table 4 Comparison of the discriminatory ability of inflammation-based prognostic scores in primary and validation cohorts

\begin{tabular}{|c|c|c|c|c|c|}
\hline Cohort & Variables & AUC & $95 \% \mathrm{Cl}$ & $P$-value & $\begin{array}{l}\text { P-value for } \\
\text { comparison to SIRI }\end{array}$ \\
\hline \multirow{12}{*}{$\begin{array}{l}\text { Primary } \\
\text { cohort }\end{array}$} & 3-year & & & & \\
\hline & SIRI & 0.795 & $0.753-0.833$ & $<0.001^{a}$ & - \\
\hline & NLR & 0.730 & $0.685-0.772$ & $<0.001^{a}$ & 0.134 \\
\hline & MLR & 0.763 & $0.719-0.803$ & $<0.001^{a}$ & $0.00 \mathrm{I}^{\mathrm{a}}$ \\
\hline & PLR & 0.669 & $0.62 I-0.7 / 4$ & $0.005^{a}$ & $0.015^{*}$ \\
\hline & MSKCC score & 0.702 & $0.577-0.827$ & $0.00 \mathrm{I}^{\mathrm{a}}$ & 0.067 \\
\hline & 5-year & & & & \\
\hline & SIRI & 0.767 & $0.723-0.807$ & $<0.001^{a}$ & - \\
\hline & NLR & 0.698 & $0.65 \mathrm{I}-0.742$ & $<0.00 \mathrm{I}^{\mathrm{a}}$ & $0.047^{\mathrm{a}}$ \\
\hline & MLR & 0.734 & $0.689-0.776$ & $<\left.0.00\right|^{a}$ & 0.241 \\
\hline & PLR & 0.611 & $0.562-0.658$ & $0.024^{a}$ & $<\left.0.00\right|^{a}$ \\
\hline & MSKCC score & 0.687 & $0.586-0.789$ & $<0.001^{a}$ & 0.118 \\
\hline \multirow{12}{*}{$\begin{array}{l}\text { Validation } \\
\text { cohort }\end{array}$} & 3-year & & & & \\
\hline & SIRI & 0.759 & $0.666-0.853$ & $<0.00 \mathrm{I}^{\mathrm{a}}$ & - \\
\hline & NLR & 0.728 & $0.655-0.794$ & $0.01 \mathrm{I}^{\mathrm{a}}$ & 0.231 \\
\hline & MLR & 0.701 & $0.626-0.769$ & $0.026^{a}$ & $0.017^{a}$ \\
\hline & PLR & 0.676 & $0.600-0.746$ & 0.051 & $0.003^{a}$ \\
\hline & MSKCC score & 0.675 & $0.507-0.843$ & 0.052 & 0.612 \\
\hline & 5-year & & & & \\
\hline & SIRI & 0.738 & $0.670-0.793$ & $<\left.0.00\right|^{a}$ & - \\
\hline & NLR & 0.673 & $0.597-0.744$ & $0.014^{a}$ & 0.224 \\
\hline & MLR & 0.688 & $0.612-0.757$ & $0.008^{\mathrm{a}}$ & 0.051 \\
\hline & PLR & 0.637 & $0.560-0.710$ & 0.052 & $0.023^{\mathrm{a}}$ \\
\hline & MSKCC score & 0.711 & $0.585-0.837$ & $0.003^{a}$ & 0.833 \\
\hline
\end{tabular}

Notes: ${ }^{a} \mathrm{AUC}$ values for SIRI and other inflammation-based prognostic scores were compared using MedCalc software. $* P<0.05$.

Abbreviations: AUC, area under the curve; MLR, monocyte-to-lymphocyte ratio; MSKCC, Memorial Sloan Kettering Cancer Center; NLR, neutrophil-to-lymphocyte ratio; $\mathrm{PLR}$, platelet-to-lymphocyte ratio; SIRI, systemic inflammation response index.

\section{Disclosure}

The authors report no conflicts of interest in this work.

\section{References}

1. Mickisch G, Carballido J, Hellsten S, Schulze H, Mensink H, European Association of Urology. Guidelines on renal cell cancer. Eur Urol. 2001;40(3):252-255.

2. Siegel RL, Miller KD, Jemal A. Cancer statistics, 2016. CA Cancer J Clin. 2016;66(1):7-30.

3. Ljungberg B, Campbell SC, Choi HY, et al. The epidemiology of renal cell carcinoma. Eur Urol. 2011;60(4):615-621.

4. Chen W, Zheng R, Baade PD, et al. Cancer statistics in China, 2015. CA Cancer J Clin. 2016;66(2):115-132.

5. Edge SB, Compton CC. The American Joint Committee on Cancer: the 7th edition of the AJCC cancer staging manual and the future of TNM. Ann Surg Oncol. 2010;17(6):1471-1474.

6. Volpe A, Patard JJ. Prognostic factors in renal cell carcinoma. World J Urol. 2010;28(3):319-327.

7. Hanahan D, Weinberg RA. Hallmarks of cancer: the next generation. Cell. 2011;144(5):646-674.

8. Labelle M, Begum S, Hynes RO. Direct signaling between platelets and cancer cells induces an epithelial-mesenchymal-like transition and promotes metastasis. Cancer Cell. 2011;20(5):576-590.

9. Cools-Lartigue J, Spicer J, Mcdonald B, et al. Neutrophil extracellular traps sequester circulating tumor cells and promote metastasis. J Clin Invest. 2013:3446-3458.

10. Mantovani A, Allavena P, Sica A, Balkwill F. Cancer-related inflammation. Nature. 2008;454(7203):436-444.
11. Pichler M, Hutterer GC, Stoeckigt C, et al. Validation of the pre-treatment neutrophil-lymphocyte ratio as a prognostic factor in a large European cohort of renal cell carcinoma patients. Br J Cancer. 2013;108(4): 901-907.

12. Chang Y, An H, Xu L, et al. Systemic inflammation score predicts postoperative prognosis of patients with clear-cell renal cell carcinoma. Br J Cancer. 2015;113(4):626-633.

13. Gunduz S, Mutlu H, Tural D, et al. Platelet to lymphocyte ratio as a new prognostic for patients with metastatic renal cell cancer. Asia Pac J Clin Oncol. 2015;11(4):288-292.

14. Qi Q, Zhuang L, Shen Y, et al. A novel systemic inflammation response index (SIRI) for predicting the survival of patients with pancreatic cancer after chemotherapy. Cancer. 2016;122(14):2158-2167.

15. Ito $\mathrm{K}$, Seguchi $\mathrm{K}$, Shimazaki $\mathrm{H}$, et al. Tumor necrosis is a strong predictor for recurrence in patients with pathological T1a renal cell carcinoma. Oncol Lett. 2015;9(1):125-130.

16. Katz MD, Serrano MF, Humphrey PA, et al. The role of lymphovascular space invasion in renal cell carcinoma as a prognostic marker of survival after curative resection. Urol Oncol. 2011;29(6):738-744.

17. Gunduz S, Mutlu H, Tural D, et al. Platelet to lymphocyte ratio as a new prognostic for patients with metastatic renal cell cancer. Asia Pac J Clin Oncol. 2015;11(4):288-292.

18. Camp RL, Dolled-Filhart M, Rimm DL. X-tile: a new bio-informatics tool for biomarker assessment and outcome-based cut-point optimization. Clin Cancer Res. 2004;10(21):7252-7259.

19. Chen Z, Shao Y, Yao H, et al. Preoperative albumin to globulin ratio predicts survival in clear cell renal cell carcinoma patients. Oncotarget. 2017;8(29):48291-48302.

20. Balkwill F, Mantovani A. Inflammation and cancer: back to Virchow? Lancet. 2001;357(9255):539-545. 
21. Coussens LM, Werb Z. Inflammation and cancer. Nature. 2002;420(6917): $860-867$.

22. Germano G, Allavena P, Mantovani A. Cytokines as a key component of cancer-related inflammation. Cytokine. 2008;43(3):374-379.

23. Lindenmann J, Fink-Neuboeck N, Avian A, et al. Preoperative Glasgow prognostic score as additional independent prognostic parameter for patients with esophageal cancer after curative esophagectomy. Eur $J$ Surg Oncol. 2017;43(2):445-453.

24. Elinav E, Nowarski R, Thaiss CA, Hu B, Jin C, Flavell RA. Inflammation-induced cancer: crosstalk between tumours, immune cells and microorganisms. Nat Rev Cancer. 2013;13(11):759-771.

25. Kuper H, Adami HO, Trichopoulos D. Infections as a major preventable cause of human cancer. $J$ Intern Med. 2000;248(3):171-183.
26. Diakos CI, Charles KA, McMillan DC, Clarke SJ. Cancer-related inflammation and treatment effectiveness. Lancet Oncol. 2014;15(11): e493-e503.

27. Ferrone C, Dranoff G. Dual roles for immunity in gastrointestinal cancers. J Clin Oncol. 2010;28(26):4045-4051.

28. Condeelis J, Pollard JW. Macrophages: obligate partners for tumor cell migration, invasion, and metastasis. Cell. 2006;124(2): 263-266.

29. Shibutani M, Maeda K, Nagahara H, et al. Prognostic significance of the lymphocyte-to-monocyte ratio in patients with metastatic colorectal cancer. World J Gastroenterol. 2015;21(34):9966-9973.
Cancer Management and Research

\section{Publish your work in this journal}

Cancer Management and Research is an international, peer-reviewed open access journal focusing on cancer research and the optimal use of preventative and integrated treatment interventions to achieve improved outcomes, enhanced survival and quality of life for the cancer patient The manuscript management system is completely online and includes

\section{Dovepress}

a very quick and fair peer-review system, which is all easy to use. Visit http://www.dovepress.com/testimonials.php to read real quotes from published authors. 